\title{
Semantic-based Print Security Information Fusion Framework for Multi-Sensor Systems in Power System
}

\author{
Hao Yang ${ }^{1, a}$, Lingling Zhang ${ }^{2, b}$, Junhua Chen ${ }^{3, c}$ \\ ${ }^{1}$ JiangXi Electric Power Research Institute, Nanchang, China \\ ${ }^{2}$ JiangXi Electric Power Research Institute, Nanchang, China \\ ${ }^{3}$ JiangXi Electric Power Research Institute, Nanchang, China \\ abirdeye@163.com, b420246384@qq.com, '10415403@qq.com
}

Keywords: multi-sensor systems; power system; information fusion; semantic; ontology; printer; information security

\begin{abstract}
The heterogeneity of information in multi-sensor systems in power system makes information fusion process more difficult, and results in divarication of information fusion. Aiming at this problem, this paper presents an automatic information fusion framework. The main contribution is the method of semantic processing in information fusion. This paper describes the definition of the overall ontology, the method of mapping local information sources of sensors to the overall ontology, the subscription and publication mechanism of semantic information, and the corresponding rules for information fusion. The framework can effectively implement automatic information fusion in multi-sensor systems in order to obtain more comprehensive, secure, accurate and fine-grained overall information.
\end{abstract}

\section{Introduction}

Multi-sensor system completely integrates lots of multi-sensor databases from different time and space, helping it obtain a more completed and unanimous information about the real scene so as to make it can analyze and decide at the last step. Because information exists in different forms and large scales in the multi-sensor system, when some urgent practical information needs to be handled promptly, a multi-sensor system that can obtain the database from different time and space is great demand then.

In order to keep watch on the facilities and working state, different sections of the power system will distribute a great number of different-typed sensors, by which related information can be obtained, fused and provided for the last decision. Each sensor collects local information of the target by the data, for example, the number and location of the failures. After finishing this, the sensor-system harmonizes with the computer to get the overall information of the target, judge whether the facility can go on working smoothly, analyze the working state of the power plant and so on according to the revised local information the sensor obtained. In view of the these, the multi-sensor system can get so accurate and timely information about the power system that it can provide reliable reason for decision and command.

The technology of information fusion has received wile application in the energy field. Currently, some main energy powers of the world have developed their own information fusion system. Multi-sensor information fusion technology is a modern information processing technology. It has such broad application in the power system as running and controlling of the power grid, monitoring the working state of the power facility, dispatching power and diagnosing technical failures and so on.

The current research mainly supposes that the multi-sensor system is homogeneity isomorphism in the process of corresponding and data collecting, therefore there is no deviation in the process of data understanding and processing. However, this kind of assumption is usually groundless when it is related with electricity. For example, under different conditions of geological situation, when different sections need to make certain change to satisfy its environment, the sensors they use and the 
information thy collect is heterogeneity. in such kind of situation, on one hand, the coordination of different sections is a dynamic process, different applied situations need different sensors, in this way, it is impossible to develope a unified information fusion system to operate. On the other hand, if the information receives no treatment, certain information deviation or loss will be resulted in. It is really an urgent problem to be solved so as to fuse the information effectively.

As for the heterogeneity of information in multi-sensor systems in power system and the deviation appeared in the process of information fusing, a semantic-based information fusion Framework is put forward so as to avoid the deviation and enforce its reliability.

With the wild application of information technology in the process of producing, manufacturing and managing of power system, the printer becomes an indispensable instrument of the offices. Most of the print control products is only supported by the software, laking certain guarantee in the sense of security. In fact, recently, printer leakage has become an unneglectable way of information security leakage.

Compared with the common information leakage, the printer leakage is hard to be traced. Confidential information of a company printed by printer may be leaked. Being lacked of the certain necessary technology, it is really very hard to find out who should be responsible.

In order to solve this problem, by using the hardware KEY as the identification, client software as the carrier and authority control as the fundamental, this paper is aimed at reaching a safe control on the printer.

\section{System Description}

The frame of the whole system can be seen Fig. 1, mainly including the overall ontology, the mapping of local information sources of sensors to the overall ontology, the subscription and publication mechanism of semantic information, and the corresponding rules for information fusion.

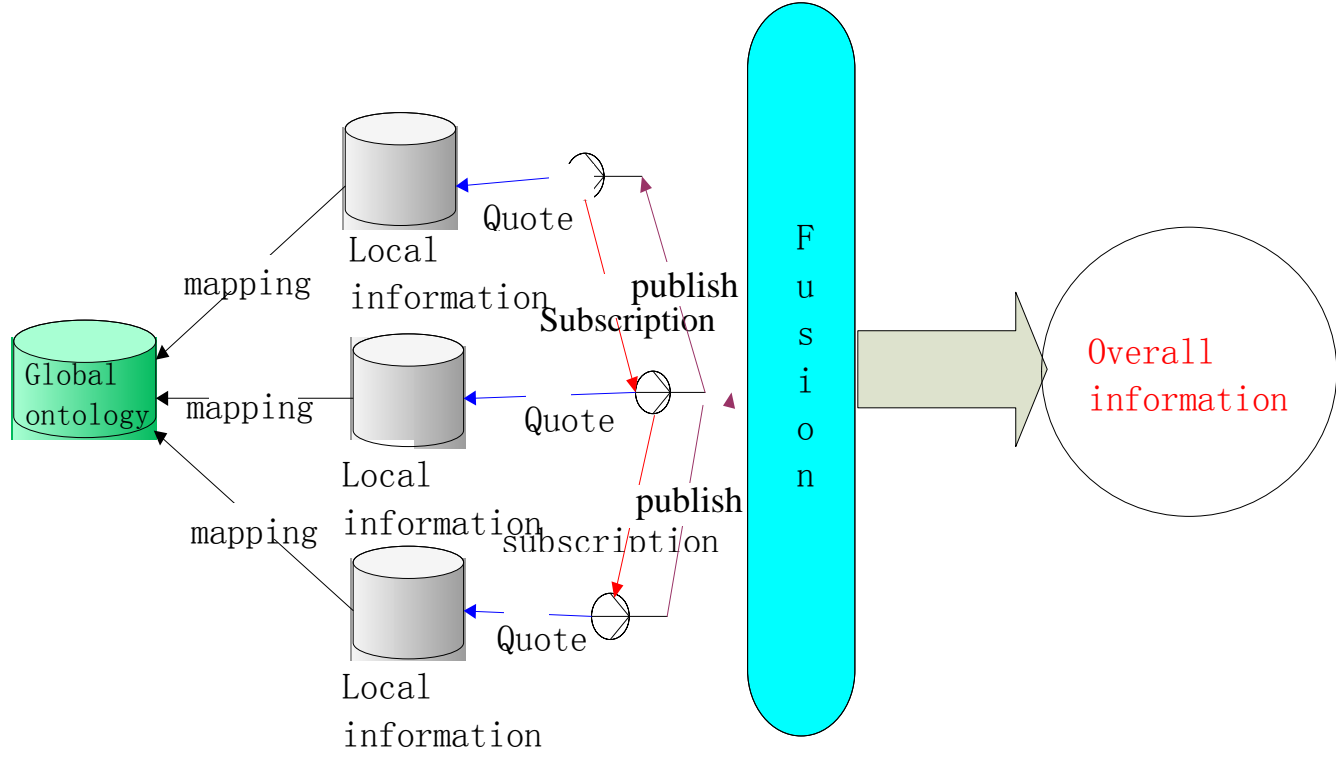

\section{Figure 1. Semantic-based information fusion frame}

Semantic ontology, Semantic ontology, which can be based to effectively erase the deviation and achieve information fusion by many sensors, is the core of the whole frame. Employing the OWL language to define semantic ontology, this frame also describes the ontology and its internal relationship between different kinds. As a language to express knowledge, OWL can fully show the semantic, and set a basis for the further information fusion. By using the OWL to define and describe information, the information defined and collected by ontology can be interpreted by machine can fused automatically by the computer, improving the efficiency in a natural way.

In the practical application of power system, hi-tech information is playing an important role, especially in diagnosing the failures and monitoring working state of power instrument. An example of semantic ontology is given below based on an simulated situation. 


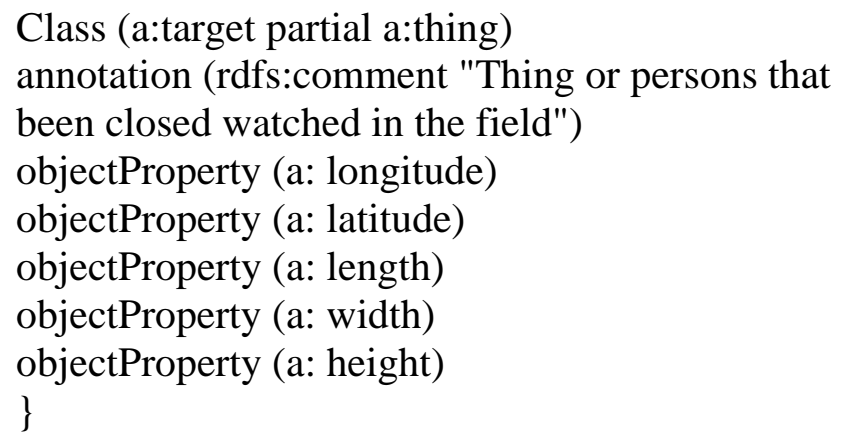

Here, a target in the power system needed to be monitored is defined. For connivence, OWL-based abstract syntax is used for the definition. In it, annotation explains the described meaning of ontology. In the following part, the object properly records respectively the corresponding properties of the monitored target, including the longitude, latitude, length, width and height and so on. These property are mainly used to describe the state of the target.

In the same way, OWL-based abstract syntax can also be used to describe the moving property of the target, such as the velocity, acceleration, azimuth, slope distance, Doppler frequency shift and so on of the target. Definition of the property can be defined as follows:

objectProperty (a: velocity)

objectProperty (a: acceleration)

objectProperty (a: azimuth)

objectProperty (a: slope_distance)

objectProperty (a: Doppler_frequency_shift)

Moreover, the other properties of the target can also be defined, such as the electromagnet radiation intensity, temperature parameter, air humidity and so on of the facilities. The form of the definition is nearly the same as the above mentioned properties.

In the power system, one of the important aims of obtaining information is to monitor the mechanical, electrical, physical, chemical variation of the targeted facility. Featured by small size, powerful functions, low energy consuming and high accuracy, the sensors can feel and monitor the above mentioned properties of each targed facility. By judging the failures or the seriousness of failures, the sensors can then determine the working state of the power system, can infuse the monitored information of the facilities into the security and dispatch system, enhancing the safety and intelligence level.

Mapping of local information sources of sensors to the overall ontology, In the power system, the multi-sensor system usually is dynamically made up of many different types of common sensors by different departments. The sensors developed by different departments collect and save information on the basis of their own stipulated l ocal data. Thus the structure and ontology used by them are usually not all the same.

In order to realize the information fusion, coordination and share with no deviation among different sensors, a unified global ontology is given in the last part of this. In this part, mapping of local information sources of sensors to the overall ontology will be introduced so as to achieve the mutual communication and share of information between different sensor systems. In this way, when the information is fused, the multi-sensors will used a common overall ontology. On one hand, it will automatically fuse the information will the least manual operation, on the other hand, the deviation in the process of information fusion will be totally erased as well.

The mapping of local information sources of sensors to the overall ontology is an important step in the process of automatic information fusion and the ontology mapping is also an important research topic. The methods of ontology mapping includes manual mapping, semi automatic mapping and automatic mapping and so on. Technically speaking, it also includes machine learning-based method, probability-based method, rule-based method and so on. The semantic-registration based semi-automatic mapping method is used in this frame. First, the overall ontology in the field is defined by the specialists in this field and the technicians in the computer to include the class of the ontology, the property of the class and the logical relation between class and class. Then, each 
department should register their own individual information source in the overall ontology, which requires to be operated by the system manager in each department. The semantic registration is dynamic, that is to say, part or total data source can be registered in accordance with their own authority. For instance, for safety, certain department will not register their whole information to the overall ontology in case certain information would be leaked out in the process of the information fusion. Once the information source is registered, their corresponding OWL document would be saved in the overall ontology server. By using the classified structure of overall ontology and class inference, the relationship between different ontology could be automatically deduced. An example is could be seen below:

Class ( a:WX217-085LLT complete intersectionOf( a: generator restriction(a:air cooler_with someValuesFrom (a: KCWQ-2400))))

Class (a:air-cooling generator complete intersectionOf (a: generator Restriction (a: air cooler_with someValuesFrom (a:air-cooling))))

Class (a: KCWQ-2400 partial a:air-cooling)

The above is a class description of air-cooling generator WX217-085LLT on the basis of OWL abstract syntax. WX217-085LLT is a kind of air-cooling generator, using air cooler KCWQ-2400 . With the help of class inference, WX217-085LLT generator is also a kind of air-cooling generator. Although this is a very simple example, in many cases, if the class interference is automatically finished, some new information will be obtained. The following part will introduce the subcription and publishing of the semantic information, as well as the important role it will play in the process of semantic information fusion.

Subscription and publishing security of the semantic information ,In the process of coordination of the multi-sensor system, in order to achieve effectively share and fuse the information, the publishing and subscription of the semantic information is needed among the sensors. By doing this, each sensor can obtain its needed information and avoid the multi-sensor system net jam, at the same time, the information can be transmitted more effectively and the width of the band can be saved. Each sensor in the multi-sensor system can subscribe and publish its needed information according to its own favor or interest.

Because the power system usually is an important security department in any country or district, the information fusion must take the security into consideration. The whole process can be described as follows: the information sensor should firstly browse the overall ontology, choose its field and becomes the subscriber. The request would then be sent to the other sensors in the same mulit-sensor system. The sensor who owns related information is called the publisher. The request usually includes: field, timespan, subscriber ID, information type and classification, and so on.

After receiving the subscribed information, the publisher should firstly check the subscriber's identity. The battle of the information war largely depends on the accurate and reliable information. In recent years, each research institute of sensor is trying to develop types of sensors to assist the collecting and transmitting of information in every possible situations. Therefore, if the coordinating information or order is intercepted and captured by the invader, expectable loss would be brought. It is really very necessary and important to fix the other's identity in the coordinating correspondence.

In order to guarantee the safe communication in the multi-sensor system, three handshakes contract in the TCP contract is used to build a transmitting way among different sensors. The reason of using such complicated identity checking model is to avoid the invading sensor disguises as the subscriber or publisher to intercept valuable information or spread false data. The whole transmitting way is as follows: first shake, after encoding, the subscriber send its own identity to the publisher to enter the send state, waiting for the reply from the publisher; the second handshake, after receiving and decoding the identity checking information, the publisher will confirm the legal identity and then send the confirmed and encoded identity information to the subscriber. If the subscriber is a systemic sensor, it will successfully decode and get a definite ID. The subscriber then send back a confirmed information with confirmed ID implying receiving. This ID is calculated by the confirmed ID on the basis of secret key. In this way, the information transmitting way is built up. 
There are seldom multi-sensor system can find the invader's invasion to the system. If the invader wants to disguise as the subscriber, it must provide the encoded identity. if it is a false identity, the publisher will soon find out the unusual and stop the communication, and spread the invasion to the whole system to warn the other sensors to protect. Even if the invader intercepts the first password and send out successfully the request, the publisher will use another set of password to encode the identity and send it to the false subscriber, it would impossible to send back the right confirmed information because it has no second set of password. Thus, it would be intercepted and caught before the second proof line. If the invader sensor disguises as the systemic publisher, when the request is sent to the false receiver, it would also not send back the right confirmed information for lacking of decoding the subscriber's encoded information. After receiving the false confirmed information, the subscriber would instantly find out the disguised invasion. According to this, the information transmitting way built up by double password and three handshakes among the sensors can effectively guarantee the information security problem in the multi-sensor system.

If the publisher sensor have any disagreement with the request from the subscriber, such as the timespan, field and so on, then the failure information including the details to be revised and adjusted will be sent back. According to the replied information, the subscriber can change the subscription and send the application again.

Printer security control, By controlling the printing source and authority to realize the safe printing, a security controlling system of the printer needs to be developed, namely combined the security checking hardware module with the KEY as the carrier with the security controlling on the printing. In this way, not only the dynamic printing can be completely showed and printing recording can be drew , refusing any external printing demand but also all the paper documents can be provided with powerful proof for their sources follow-ups.

The controlling system mainly covers the following 4 subsystems:

1) Safety monitoring system;

2) KEY information writing tool;

3) Clients reside monitoring subsystem ;

4) KEY information security module.

\section{System Implement}

After each sensor in the multi-sensor system collecting and obtaining certain related local information, the local information will be transformed into semantic data expressed by the OWL language on the basis of the mapping relationship between local information source and the overall ontology. According to the request from the subscriber sensor to the publisher sensor, the packed semantic data package will be sent. After receiving the corresponding information, the subscriber sensor will firstly make an analysis. Because these information are based on the same ontology, they can smoothly build up a correlation among them so as to fuse the information. Due to the different location and azimuth of the sensors, it is very hard to get a totally identical information. In view of this, the following rules are defined to fuse the information whin this framework:

rule 1, When sensor A finds the information of certain field in the target and sensor B finds no information, then, the local information detected by sensor A can be used as the overall information in the field of target.

rule 2,When sensor $A$ and sensor $B$ finds certain related information in the same field of the target, if sensor A takes a more connivent location than sensor B for observation, then the information provided by sensor $A$ is treated as the overall information.

rule 3,. When all the field detected by many sensors are all incomplete, all the local information of different fields will be fused to obtain the overall information of the target.

rule 4, When lots of sensors collect different information from the same field, and rule 2 and rule 3 are not sufficient to make any difference, then the information collected by sensor of higher reliability in the multi-sensor system can be used as the overall information of the target.

rule 5,Suppose the sensor A collects field c1, and sensor B collects c2, and c1 is the subcollection of c2, then the information collected by sensor A is regarded as the overall information of the target. 
In the multi-sensor system, different sensors can fuse different local information effectively on the basis of the above mentioned 5 rules by citing the common domain ontology. Fully taking the location, field, reliability and class into considerationl, the fusion rules can fuse the information of multi-sensor system with high efficiency.

As for rule 1, when certain information can only be obtained by specific sensors (for example, some sensors are equipped with some highly smarted information collecting device) rather than the others, then, the information obtained by sensors can be used.

As for rule 2, in many cases, many sensors all can get certain information about the target. However, there is definitely some difference between them because of the different location and azimuth of each sensor. Take the temperature for the example, the closer to the target, the closer to the real temperature of the target and the farther, the greater the difference. Therefore, when the local information from the same field by many sensors are not totally identical, the information from the closer sensor or the closer of the best azimuth to the target is usually chosen.

As for rule 3, different types of sensors have different focus on the information they can obtain. For the same target, different sensors can usually provide information of specific field. By collecting all the information of the overall ontology sent by different sensors, the overall information of the target can then be got.

As for rule 4, for the same information filed, if the information sent back by different sensors are not identical and are not in accordance with the rule 2 and rule 3, the rule 4 is therefore set. That is, the information sent by sensors of high reliability can be treated as the overall information of the target, because the information from these sensors are usually more reliable.

As for rule 5, overall ontology is a layered definition. When a information field covering the information obtained by a sensor is a subcollection of another information domain covering the information by another sensor, the sensor which obtains the subcollection field is regarded as the overall information, because the information obtained in this way is usually more fine-grained.

Safety monitoring system, B/S technology is used to realize the printer safety information management and vivid display. For the control of the internal workers of the system, only the authorized ones can use the printer.

Specific technicians are authorized to set the KEY so as to realize the user access distribution about the KEY information writing tool.

Realize the recording and inquiry of the setting of the KEY so as to keep track of all the KEY.

KEY information writing tool ,A C/S model-based KEY setting program for printer security control help the client to input their registration informtion, security information into the KEY, making the KEY registration become the hardware part of the printer security control module.

Clients reside monitoring subsystem, A C/S model-based clients reside monitoring program can be developed. Besides upgrading automatically, the program should also meets the following requirements:

Actively detect all the printing by any client and make the recording about any printing, such as the KEY number, registration name, name of the printed document, time of the printing, ID and name of the printing and so on.

Set restriction to any printing lacking of the security KEY, printing is not permitted and the action should be recorded.

Only the user with the security KEY is permitted to print, and the time, location of setting the security KEY as well as the registration information should be recorded.

KEY information security module, By using the KEY hardware as the carrier, the embedded registration information and password security information will be read and detected its related authority. Thus, the registration information in the KEY can be chected and the embedded password information can be checked at the same time as well.

\section{Conclusion}

With the wide application of information technology in the power system, the multi-sensor system is becoming more and more important in energy field. In view of the heterogeneity of information in 
the information fusion process in multi-sensor system, a semantic-based information fusion framework is put forward to effectively get rid of the information deviation in the process of information fusion in the multi-sensor system, avoid invading and obtain a more complete, accurate and fine-grained overall information. By drawing the printing record and refuse any unauthorized printing demand, the paper document can be traced, thus the security of the system information is highly improved as well.

\section{References}

[1] Wang Wenzhi, An analysis on the applied prospect of multi-sensor information fusion technology in the power system[J]. Electric Power Construction, 2003,10:56-58.(in Chinese)

[2] Li Y. Multi-Sensor Information Fusion and its Application[C]. International Conference on Wireless Communications, Networking and Mobile Computing, 2007, pp.6095-6098.

[3] Li Quanxi, Gong Qingmin. An applied research on the information fusion in the greenhouse watermelon growing[J], Microcomputer Information, 2009,16. (in Chinese)

[4] Li Bin,Zhang Weiguo.Information Fusion Based on Self-organizing Neural Network in Fault Diagnosis[J].journal of Transduction Technology,2006,19(6):2567-2570.(in Chinese)

[5] Li Gang, Zeng Ruili Ding Ru Lin Ling.Research on City Traffic Monitoring and Controlling System Based on Multi-Information Fusion Technique[J]. Journal of Transduction Technology,2006, 19(6): 2707-2711.(in Chinese)

[6] Qi Yi, Peng Hong, Wang Tao. Study on Mine Fire Detection System Based on Fuzzy Information Fusion [J]. Colliery Mechanical \& Electrical Technology, 2009, 3. (in Chinese)

[7] Luo Zhizeng, Yeming. Bayes based method of multiple sensory information fusion algorithm and its application [J]. Journal of Transduction Technology, 2001,14(3):210-215. (in Chinese)

[8] He You, Lu Da, Peng Yingyu, Wang Guohong. The information fusion and application of multi-sensor[M]. 2000, Publishing House of electronics industry. (in Chinese)

[9] Nie Guihua, Zuo Xiuran, Chen Donglin. An improved concept similarity computing method in ontology mapping [J]. Computer applications, 2008,28(6):1563-1565. (in Chinese)

[10] Tang Jie, Liang Bangyong, Li Juanzi, Wang Kehong. Automatic mapping of ontology in the semantic web[J]. Computer Magazine, 2006,29(11):1956-1976. (in Chinese)

[11] Li Guanyu, Shi Yimin, Semantic Web ontology mapping based on machine learning research [J]Microcomputer Information, 2009,25(9). (in Chinese)

[12] Li Guofang. Ontology mapping based on Bayesian network model[D], Wuhan University, 2006. (in Chinese)

[13] Jiang Chao, Nie Peirao, Xu Ruzhi. An ontology based on graph model and heuristic rules of complex ontology mapping method[C]. The eleventh national enterprise information and Industry Engineering Conference, 2007. (in Chinese)

[14] OWL Web Ontology Language Overview [EB/OL].http://www.w3.org/TR/owl-features/

[15] OWL Reasoning Examples[EB/OL]. http://owl.man.ac.uk/2003/why/latest/ 